\title{
Razonamiento moral entre estudiantes de Ingeniería en Sistemas: conflictos éticos sobre Tecnologías de la Información
}

\section{Moral reasoning among Systems Engineering students: ethical conflicts over Information Technology}

\author{
Andrés M. Mora-Ortiz, Pablo A. Rivas-Franco y Marie-France Merlyn-Sacoto \\ Pontificia Universidad Católica del Ecuador
}

\section{Resumen}

El presente estudio tuvo como objetivo explorar la posible relación entre los procesos de razonamiento moral (emocionales y racionales) y la resolución dada a los conflictos éticos en relación a las Tecnologías de la Información (TI). Para ello, se trabajó con una muestra de 177 estudiantes de Ingeniería de Sistemas (83\% hombres, $17 \%$ mujeres) de entre 17 a 26 años, a quienes se les aplicó el 'Ethical IT Survey' y un cuestionario suplementario para medir razonamiento moral. Los resultados indican que existe diferencias en los puntajes obtenidos para las cuatro categorías de escenarios analizados, ubicando a las respuestas de los temas de Privacidad y Propiedad como éticamente cuestionables, y a los de Precisión y Accesibilidad como antiéticas. El tipo de razonamiento que prevalece es el emocional. Asimismo, más de la mitad de los participantes reportan haber cometido conductas antiéticas en lo que respecta a las categorías de Propiedad y Accesibilidad, lo que muestra una discrepancia entre la apreciación cognitiva del conflicto ético y la ejecución de conductas relacionadas con estas temáticas. Se concluye que es fundamental en la formación de los estudiantes enfatizar la importancia de la ética en los asuntos relacionados con las TI.
\end{abstract}

Palabras clave: Razonamiento moral, Tecnologías de la Información, Ética

\begin{abstract}
This study investigated the relationship between the processes of moral reasoning (emotional and rational) and the way ethical conflicts were solved in relation to Information Technology (IT). To this end, a sample of 177 Systems Engineering students ( $83 \%$ men, $17 \%$ women) aged 17 to 26 years completed the 'Ethical IT Survey' and a questionnaire to measure moral reasoning. The results show differences in the scores obtained for the four categories of scenarios analyzed, placing the responses to the topics of Privacy and Property in the ethically questionable category and those of Precision and Accessibility within the unethical category. The dominant type of reasoning was emotional. Furthermore, more than half of the participants reported having engaged in unethical behavior regarding the categories of Property and Accessibility, suggesting a discrepancy between the cognitive appreciation of the ethical conflict and engaging in behaviors related to these issues. In conclusion, we suggest that the training of such students should emphasize the relevance of ethics regarding IT-related issues.
\end{abstract}

Key words: Moral reasoning, Information Technology, Ethics

Cómo citar este artículo: Mora-Ortiz, A. M., Rivas-Franco, P. A y Merlyn-Sacoto, M. F. (2020). Razonamiento moral entre estudiantes de Ingeniería en Sistemas: conflictos éticos sobre Tecnologías de la Información. Escritos de Psicología - Psycholgical Writings , 13 (2), 60-70. https://doi.org/1024310/espsiescpsi.v13i2.12410

Correspondencia: Marie-France Merlyn Sacoto, Av. 12 de octubre 1076 y Roca- Facultad de Psicología- Pontificia Universidad Católica del Ecuador. Quito, Ecuador. mfmerlyns@puce.edu.ec. Coautor Andrés Mauricio Mora Ortiz, andresm.mora94@ gmail.com. Coautor Pablo Andrés Rivas Franco pablo_rivas1905@hotmail.com 


\section{Introducción}

El estudio del razonamiento moral es un tema común en la historia de la psicología. Autores icónicos y fundamentales en psicología cognitiva, como Piaget (1935) y Kohlberg (1976) lo han abordado como producto de la ontogenia del ser humano (Hersh, Reimer y Paolitto, 2002). Posteriores aproximaciones ampliaron el estudio del razonamiento y la moralidad como fenómenos psicológicos, a través de diversidad de propuestas desde la filosofía, así como la psicología social, cognitiva, y neurocognitiva (Evans y Stanovich, 2013). Uno de los abordajes más vigentes y contemporáneos corresponde a las teorías de proceso dual, que sostienen que el razonamiento puede ser explicado mediante dos procesos cognitivos: uno automático, veloz, y basado en experiencia previa; y otro deliberado, lento y consecuente al contexto (Evans y Stanovich, 2013). Entre dicho conjunto de teorías, destaca la propuesta reciente realizada por Greene (2014a; 2014b; 2015), quien formula una teoría de proceso dual específicamente para el razonamiento moral. Para este autor, el razonamiento moral corresponde a la elaboración de conclusiones alrededor de aquellos asuntos que se reconocen como morales (Greene, 2014a; 2015). Y se puede realizar a través de los dos procesos anteriormente descritos, que él denomina respectivamente como emocional y racional (Greene, 2014a; 2014b; 2015). El autor plantea la siguiente tendencia: el razonamiento emocional —automático, veloz — está usualmente vinculado a la consideración de los valores en juego alrededor de una acción moral, siendo deontológico; mientras que el razonamiento racional - controlado, lento- estaría vinculado a la consideración de las consecuencias prácticas de una acción moral, siendo consecuencialista (Greene, 2007; 2014a). Se trata, por lo tanto, de dos disposiciones psicológicas innatas a la neurocognición del ser humano: la emocional siendo más eficaz — por ser automática al estímulo y responder a partir de valores ya aprendidos-, y la racional más flexible - por ser deliberada respecto al estímulo y responder considerando diversidad de fuentes disponibles- Greene, 2007, 2014a; 2014b).

El respaldo de la teoría de Greene proviene de una investigación preliminar sobre dos dilemas morales: el «dilema del tranvía (véase el anexo)» y el «dilema del puente (véase el anexo)». Similares en su estructura, plantean situaciones en donde se debe arriesgar la vida de una persona para salvar las de cinco. Sin embargo, en el «dilema del tranvía» esto se hace de manera indirecta, mientras que en el del puente, se debe dar muerte directamente. La mayoría de personas responde que sacrificaría la vida de una persona dentro del primer dilema, donde la muerte es indirecta, y no en el segundo caso, donde se da muerte directamente (Greene, Sommerville, Nystrom, Darley y Cohen, 2001; Greene, Nystrom, Engell, Darley y Cohen, 2004). El autor aduce que la divergencia de respuestas a los dilemas se encuentra a nivel cognitivo, en la inclinación del «dilema del puente» para involucrar reacciones emocionales que el «dilema del tranvía» no puede: la idea de empujar a alguien hacia la muerte de manera directa genera reacciones emocionales mucho más prominentes que la idea de activar una palanca y generar la misma muerte de manera indirecta (Greene et al., 2001; Greene, et al., 2004). Posteriormente, a través de estudios de neuroimagen (Greene et al., 2001; Greene et al., 2004), se comprueba además que las áreas cerebrales activadas de los participantes durante la consideración del par de dilemas presentados son diferentes: durante la consideración del «dilema del puente», se midieron reacciones más cortas y con activación del córtex prefrontal ventromedial, área asociada con conducta automática y respuestas de reflejo y emocionales; en comparación al «dilema del tranvía», que obtuvo reacciones más largas y con activación de la corteza prefrontal dorsolateral, área asociada con demanda de esfuerzo a la memoria de trabajo y respuestas que requieren de raciocinio. Otros estudios amplían y corroboran estos datos (Greene et al., 2001; Greene et al., 2004).

Si bien el razonamiento moral suele ser investigado en laboratorio, es un proceso inherente a la cognición humana: Bucciarelli, Khemlani y Johnson-Laird (2008) lo definen como un proceso cognitivo donde el individuo diferencia sus consideraciones morales, donde distingue, por tanto, lo que es aceptable para sí mismo, de lo que no lo es. Este proceso suele ser estudiado a través de los "dilemas morales" (como los planteados anteriormente), que son situaciones ambiguas en donde varios valores entran en conflicto, y que el individuo debe resolver. En su vida cotidiana, las personas también se ven confrontadas a este tipo de dilemas: muchas situaciones desafían la moralidad del ser humano, tanto en su vida privada como en la profesional.

Así, por ejemplo, en la actualidad, el uso de las Tecnologías de la Información (TI) supone una fuente amplia de dilemas morales. Se trata de aquellas tecnologías capaces de codificar digitalmente información: abarcan a los ordenadores, teléfonos móviles, plataformas virtuales, hasta llegar a la inteligencia artificial y la robótica (Sullins, 2018). Son contemporáneas, han alcanzado un desarrollo exponencial sin precedentes, y han transformado - y lo siguen haciendo- de manera permanente las sociedades humanas. Dadas estas características, han traído consigo una serie de problemas éticos sin precedentes también (Reynolds, 2014; Stahl, Timmermans y Flick, 2016; Schultz, 2006; Sullins, 2018). 
Partiendo de la dificultad para conceptualizar tas TI en sí, se han propuesto una serie de modelos para esquematizar el conjunto de dilemas éticos que suponen: se han realizado clasificaciones según el tipo de tecnología, el área de impacto, los actores involucrados, la función en juego, entre otros (Reynolds, 2014; Stahl, et al. 2016; Schultz, 2006; Sullins, 2018). Una de las clasificaciones más antiguas, empero más vigentes, es la realizada por Mason (1986): el marco conceptual PAPA. Este abordaje plantea que, más allá del desarrollo tecnológico que puedan experimentar las TI, en su relación con el manejo de información, siempre se pueden encontrar cuatro categorías éticas fundamentales: «Privacidad», «Precisión», «Propiedad», y «Accesibilidad» —o PAPA, por sus siglas en inglés- (Mason, 1986). Cada categoría se sustenta en una reflexión ética respecto del manejo de la información bajo las TI. La primera, «Privacidad», reflexiona sobre qué información puede un individuo mantener privada o no, cómo hacerlo, y quiénes y con qué fines están involucrados. La siguiente categoría, «Precisión», reflexiona respecto de quién es responsable de la validez y autenticidad de la información, así como sus posibles errores. La tercera categoría, «Propiedad», se refiere a los derechos intelectuales de la información: quién es propietario de la información y sus canales de distribución. Y, finalmente, la última categoría, «Accesibilidad» se cuestiona por quién tiene capacidad, derecho y/o autoridad para acceder a qué información, y bajo qué garantías o condiciones (Brown, 2018; Martín y Woodward, 2011; Mason, 1986; Woodward, Martin e Imboden, 2011). La ventaja del marco conceptual PAPA yace en su amplitud para abarcar la diversidad de TI y sus problemáticas en cuatro categorías simplificadas. Varios estudios han utilizado la propuesta de Mason y la han probado vigente en la actualidad (Brown, 2018; Peslak, 2006; Woodward, et al., 2011). De hecho, este enfoque es ampliamente utilizado para la investigación (Ming, Jabar, Sidi y Wei, 2015).

Los esfuerzos sociales-desde la academia, la sociedad civil, la industria, el gobierno-por comprender estas tecnologías y sus impactos, y, así, regularlas, son superados constantemente por la complejidad y avance de las mismas. Sin protocolos universales ni antecedentes histórico-sociales claros, los profesionales involucrados en su desarrollo se ven obligados a tomar decisiones de carácter moral por sí mismos, frente a situaciones ético-morales sin referencias previas (Harris, Yang, Yates y Kruck, 2011; Reynolds, 2014; Stahl y Rogers, 2013; Sullins, 2018). Por lo tanto, cabe la pregunta: ¿cómo razonan moralmente estos profesionales, dentro de su campo laboral, al momento de enfrentar conflictos éticos provenientes de las TI?

El objetivo de la presente investigación es, justamente, analizar los procesos de razonamiento moral que realizan los mencionados profesionales en formación para la resolución de conflictos éticos relacionados a las TI. Se explora la posible relación entre los procesos de razonamiento moral empleados y la resolución dada a los conflictos éticos en relación a dichas tecnologías.

\section{Participantes}

\section{Método}

Se seleccionó como participantes a potenciales futuros profesionales asociados al campo de las TI: los estudiantes de Ingeniería en Sistemas de la Pontificia Universidad Católica del Ecuador (PUCE). De esta población, con un total de 297 estudiantes para febrero de 2019, se calculó, considerando un intervalo de confianza del 95\%, una muestra representativa mínima de 168 estudiantes para obtener datos estadísticamente significativos. La muestra final de participantes constó de un total de 177 estudiantes. Como criterios de inclusión se consideraron: cualquier estudiante de cualquier semestre de la carrera que al momento la esté cursando, un rango de edad entre 17 a 26 años de edad, y el consentimiento voluntario de participar en la investigación. Como criterios de exclusión, se consideraron: estudiantes que ya hayan obtenido un título en otra carrera, y que voluntariamente declaren cualquier patología psicológica diagnosticada profesionalmente. Los 177 estudiantes participaron de manera voluntaria a condición de ser garantizado su anonimato: solo se obtuvieron datos generales sobre su edad y género; ningún otro dato de identidad está disponible. Todos los participantes se encuentran dentro del rango de edad estipulado (17-20 años: $43.50 \%$; $20-24$ años: 46.33\%; $25-26$ años: $10.17 \%$ ). Respecto al género, se evidenció una gran diferencia estadística: $83 \%$ hombres vs. $17 \%$ mujeres, razón por la cual no se realizan comparaciones entre grupos por género en los análisis.

Con el fin de garantizar el cumplimiento de normas éticas pertinentes, todo el proceso investigativo fue realizado bajo la autorización y supervisión del Comité de Ética en Investigación en Seres Humanos de la PUCE (CEISH-PUCE), así como la colaboración expresa de la Facultad de Ingeniería. 


\section{Instrumentos}

Se aplicaron dos instrumentos: la herramienta 'Ethical IT Survey' (2017) de Albert Harris y un cuestionario suplementario a esta. El instrumento de Harris (2017) constituye un cuestionario cuantitativo, sostenido teóricamente por el marco conceptual PAPA de Mason (1986). Su objetivo principal es explorar la opinión del participante sobre temas éticos relacionados a las TI. Consiste de un total de 22 escenarios -y un total de 36 ítems, pues algunos escenarios tienen más de un ítem de pregunta-, los cuales presentan dilemas éticos relacionados con las $\mathrm{TI}$ y clasificados según las cuatro categorías PAPA. Cada ítem describe una conducta hipotética, pero plausible, en que un personaje realiza una acción, alrededor de las $\mathrm{TI}$, que podría resultar éticamente conflictiva. Los participantes califican el ítem según su grado ético, mediante una escala Likert de cinco puntos: (0) conducta ética, (1) aceptable, (2) cuestionable, (3) antiética y (4) delictiva. Al culminar los 22 escenarios, los participantes responden a diez preguntas de experiencia, con respuestas de tipo sí o no, también basadas en las categorías PAPA, donde indican el haber cometido, o conocer a alguien que haya cometido, cierto comportamiento éticamente conflictivo en cuanto a las mencionadas tecnologías. Al finalizar, los participantes responden dos preguntas extra, en que reportan, a través de una escala de Likert, la importancia que le dan al campo de la ética relacionada con las $\mathrm{TI}$ y el conocimiento que tienen de estas. La confiabilidad del instrumento fue medida a través del alfa de Cronbach, que en este caso fue de 0.782 , lo cual se considera adecuado. Para su utilización, se solicitó el permiso correspondiente al autor del test.

En cuanto al cuestionario suplementario, este fue aplicado simultáneamente al instrumento de Harris. Se trata de una herramienta cualitativa, desarrollada para esta investigación, que apunta a demostrar el razonamiento moral empleado por los participantes al evaluar los escenarios, a través de la teoría de Greene (2014a; 2014b; 2015). El cuestionario consta en su totalidad de la pregunta abierta "Justifique brevemente su respuesta», donde se pide a los participantes explicar, una por una, la calificación emitida a todos los 36 ítems, mientras se los va resolviendo. El objetivo de este cuestionario es indagar el razonamiento que el participante ha utilizado para emitir su calificación sobre el ítem. Se emplea una codificación por contraste fundamentada en los indicadores teóricos que Greene (2007; 2014a; 2014b) dispone para diferenciar los dos razonamientos: para identificar razonamiento emocional se buscan respuestas deontológicas — basadas en la declaración de un derecho o deber-, y para razonamiento racional se buscan respuestas consecuencialistas — basadas en argumentación en pos de los efectos de la acción-. Cabe agregar que este cuestionario fue añadido a la misma 'Ethical IT Survey' (2017) en su aplicación virtual gracias a la aprobación y colaboración de Harris. Tras finalizar la calificación y justificación simultánea de los 22 escenarios, se continúa con las diez preguntas de sí o no; y, finalmente, las dos preguntas extra sobre la importancia de la ética en consideración de TI y el conocimiento sobre estas. De esta manera, la aplicación total de todos los apartados toma a los participantes aproximadamente treinta minutos a una hora.

\section{Procedimiento}

Ya delimitada la muestra, se pidió la colaboración de la Facultad de Ingeniería-PUCE, a través de la Dirección de Carrera de Ingeniería en Sistemas, así como de los docentes y los estudiantes, para habilitar la aplicación electrónica de los mencionados instrumentos. Una vez habilitada, se pidió la colaboración de los estudiantes, de manera totalmente voluntaria y anónima, en la aplicación simultánea de los mencionados instrumentos -'Ethical IT Survey' de Harris (2017) y el cuestionario suplementario- vía electrónica, a través de la plataforma virtual Qualtrics. Para ello, el participante siempre, automáticamente, recibió una página inicial en la cual constaba el consentimiento informado virtual, así como el instructivo de los instrumentos. La aplicación para reunir la muestra mínima se hizo entre mayo y septiembre de 2019. Al culminar la recolección de datos, se cerró el periodo de participación y la aplicación de los instrumentos y se comunicó al autor del test (Harris) al respecto, con el fin de dar por concluido el uso del test y solicitar los datos obtenidos, almacenados en sus servidores, para su respectivo procesamiento. Este constó de dos fases: una primera de filtración de datos, en que se revisaron las aplicaciones de los participantes, con el fin de retener solo aquellas válidas; y una segunda de análisis estadísticos, para la cual se utilizó el procesador SPSS versión 25. 


\section{Resultados}

Puntuaciones de la muestra en los diferentes escenarios

El test aplicado plantea 22 escenarios que corresponden a las categorías de: Privacidad (5 escenarios), Precisión (5), Propiedad (7) y Accesibilidad (5). Se calculó el puntaje promedio para cada uno de los escenarios, para luego clasificarlos y calcular a su vez un promedio por tema. Los resultados obtenidos se exponen en la Tabla1.

Tabla 1

Medias obtenidas por escenarios y por temas principales

\begin{tabular}{|c|c|c|c|c|c|c|}
\hline \multirow[b]{2}{*}{ Categorías } & \multicolumn{3}{|c|}{ Estadísticos por Escenarios } & \multicolumn{3}{|c|}{ Estadísticos por Categorías } \\
\hline & İtems/ escenario & Media & D.T. & Media & D.T. & Rango promedio \\
\hline \multirow{8}{*}{ Privacidad } & $1 a$ & 0.79 & 0.91 & 1.79 & 1.20 & 1.95 \\
\hline & $1 \mathrm{~b}$ & 2.50 & 0.81 & & & \\
\hline & 10a & 2.61 & 0.78 & & & \\
\hline & $10 \mathrm{~b}$ & 1.28 & 1.18 & & & \\
\hline & 14 & 1.80 & 1.05 & & & \\
\hline & 16 & 1.36 & 1.11 & & & \\
\hline & 18a & 1.34 & 1.19 & & & \\
\hline & $18 \mathrm{~b}$ & 2.63 & 0.82 & & & \\
\hline \multirow{6}{*}{ Precisión } & 2 & 3.31 & 0.93 & 2.95 & 1.05 & 3.06 \\
\hline & 5 & 3.23 & 0.73 & & & \\
\hline & $9 a$ & 2.07 & 1.06 & & & \\
\hline & $9 b$ & 3.46 & 0.82 & & & \\
\hline & 11 & 3.08 & 0.78 & & & \\
\hline & 13 & 2.58 & 1.18 & & & \\
\hline \multirow{11}{*}{ Propiedad } & 3 & 2.69 & 0.97 & 2.23 & 1.23 & 2.40 \\
\hline & 4 & 1.95 & 0.98 & & & \\
\hline & 6 & 1.54 & 1.04 & & & \\
\hline & $7 a$ & 1.62 & 0.98 & & & \\
\hline & $7 \mathrm{~b}$ & 2.63 & 0.77 & & & \\
\hline & 20 & 3.58 & 0.80 & & & \\
\hline & 21a & 2.94 & 1.01 & & & \\
\hline & 21b & 3.01 & 0.94 & & & \\
\hline & $22 a$ & 0.73 & 0.79 & & & \\
\hline & $22 \mathrm{~b}$ & 1.86 & 1.05 & & & \\
\hline & $22 c$ & 1.96 & 1.01 & & & \\
\hline \multirow{6}{*}{ Accesibilidad } & 8 & 1.66 & 0.98 & 2.47 & 1.14 & 2.59 \\
\hline & 12 & 1.62 & 0.85 & & & \\
\hline & 15 & 2.86 & 1.00 & & & \\
\hline & $17 a$ & 2.81 & 0.97 & & & \\
\hline & $17 \mathrm{~b}$ & 3.23 & 0.90 & & & \\
\hline & 19 & 2.63 & 1.03 & & & \\
\hline
\end{tabular}

Nota: $n=177$

Para verificar si existen diferencias entre las puntuaciones obtenidas para las cuatro categorías se aplicó la prueba no paramétrica $W$ de Kendall para muestras relacionadas que compara los rangos promedios. El coeficiente obtenido es $W=0.154$ ( $p>.000)$, lo que muestra que sí existen diferencias entre los puntajes de las cuatro categorías.

Establecida la diferencia, las medias pueden entonces ser ubicadas dentro de lo que representan según la escala del test, que plantea que: los puntajes entre $0-0.8$ son considerados éticos, entre 0.9 - 1.6 son aceptables, entre 1.7 - 2.4 cuestionables, entre 2.5 - 3.2 se consideran antiéticos y de 3.4 - 4, delictivos. Las medias obtenidas ubican a las respuestas de los temas de Privacidad $(M=1.79)$ y Propiedad $(M=2.23)$ como cuestionables, y las medias de Precisión $(M=2.95)$ y Accesibilidad $(M=$ 2.47) como antiéticas.

Tipo de razonamiento moral empleado

Para evaluar el tipo de razonamiento moral (racional o emocional), los investigadores calificaron por separado la justificación de las respuestas dadas por cada uno de los participantes. Sus evaluaciones fueron comparadas usando la prueba Kappa de Cohen para cada uno de los escenarios. 
Tabla 2

Acuerdo entre jueces para la clasificación de respuestas según el tipo de razonamiento moral

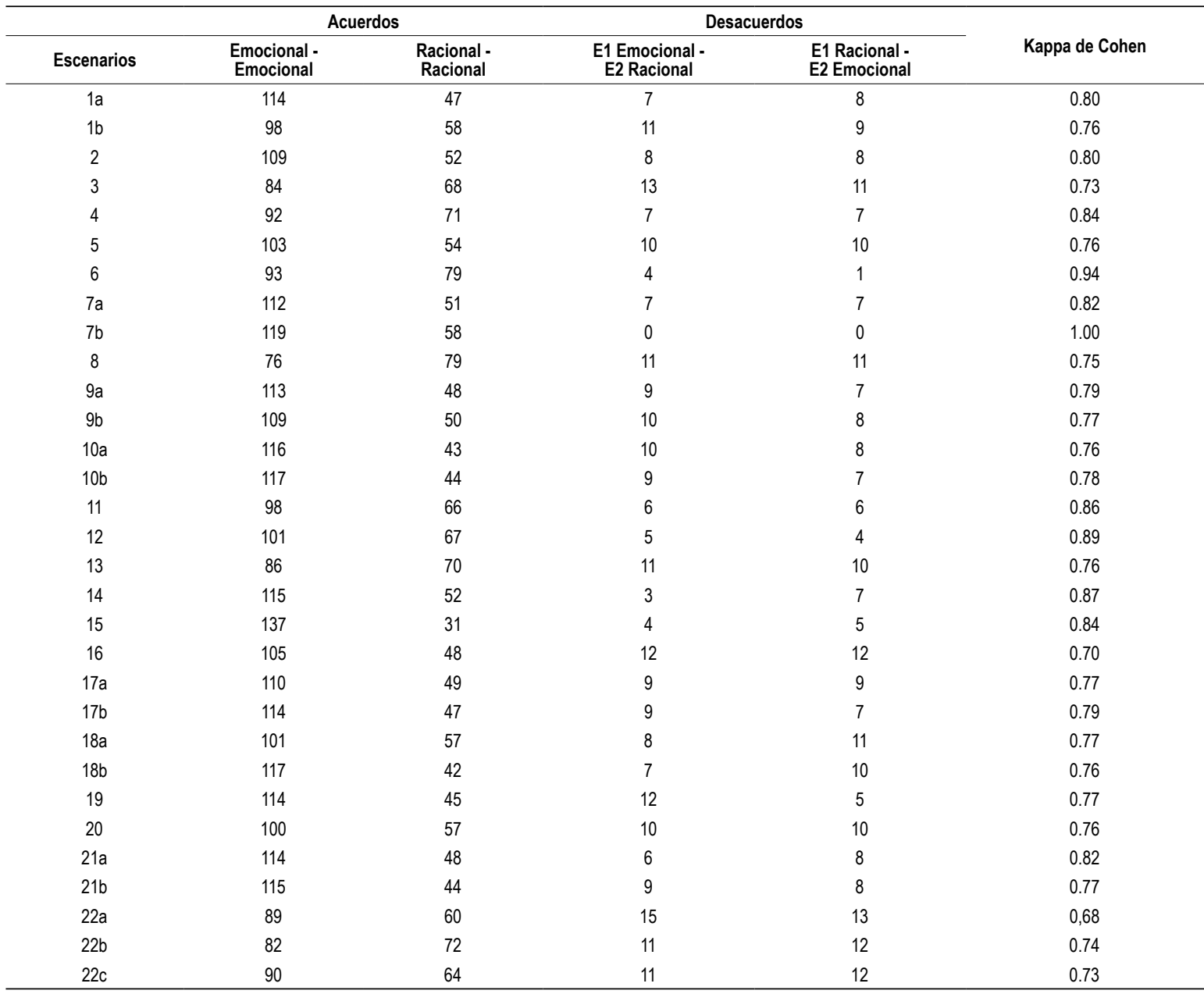

Nota: $p<.000$

La Tabla 2 muestra los resultados sobre acuerdos y desacuerdos entre ambos evaluadores. Los niveles de Kappa oscilan entre 0.68 y 1 , lo que muestra que el acuerdo entre jueces está entre "fuerte" y "casi perfecto" (Dubé, 2008).

Una vez establecido el acuerdo entre jueces, se procedió a resumir las calificaciones de acuerdo obtenidas entre ambos evaluadores para los razonamientos emocional y racional, para así poder determinar cuál tipo de razonamiento primaba para cada una de las diferentes categorías PAPA (ver Tabla 3).

Tabla 3

Temas PAPA por tipo de Razonamiento Moral

\begin{tabular}{lcccccccc}
\hline & \multicolumn{2}{c}{ Privacidad } & \multicolumn{2}{c}{ Precisión } & \multicolumn{2}{c}{ Propiedad } & \multicolumn{2}{c}{ Accesibilidad } \\
\cline { 2 - 8 } & Número & Porcentaje & Número & Porcentaje & Número & Porcentaje & Número & Porcentaje \\
\hline Emocional & 883 & 69.31 & 618 & 64.51 & 1090 & 61.86 & 652 & 67.22 \\
Racional & 391 & 30.69 & 340 & 35.49 & 672 & 38.14 & 318 & 32.78 \\
Total & 1274 & & 958 & & 1762 & & 970 & \\
\hline
\end{tabular}

La Figura 1 evidencia que en todas las categorías el tipo de razonamiento que prevalece es el emocional. 
Figura 1

Porcentajes de razonamiento moral emocional y racional por categorías PAPA

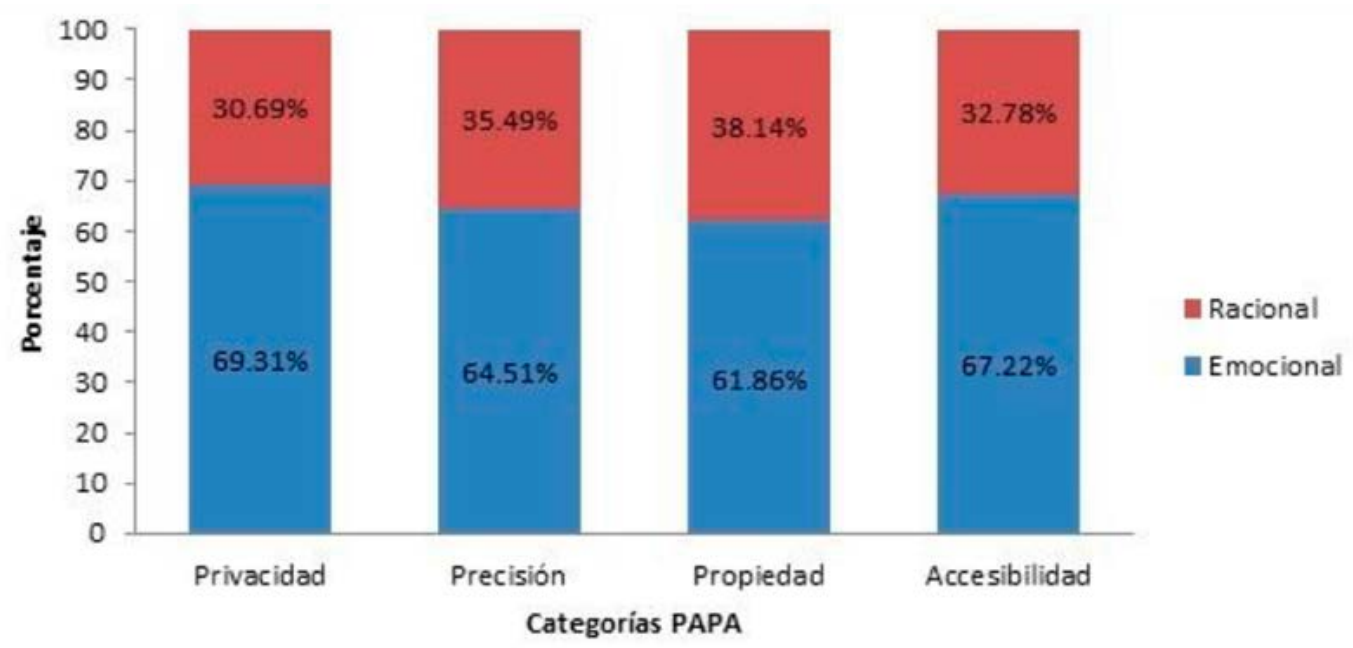

\section{Conductas antiéticas realizadas}

En cuanto a las diez preguntas sobre actos éticamente incorrectos realizados o no, dentro de cada categoría PAPA, los resultados muestran que en las categorías de Privacidad y Precisión no se han cometido en su mayoría este tipo de infracciones; por otra parte, se constata que más de la mitad de los participantes sí han cometido conductas antiéticas en lo que respecta a las categorías de Propiedad (59\%) y Accesibilidad (58\%) (Figura 2 ).

\section{Importancia percibida de la ética y el conocimiento sobre las TI}

Finalmente, se evaluó la importancia que los participantes otorgan a la ética relacionada a las TI, desde el ámbito estudiantil hasta el profesional, así como el conocimiento que reportan poseer sobre las mismas.

En cuanto a la ética, la mayoría de los participantes (83\%) ubican su importancia únicamente en el ámbito profesional, dejando de lado el interés por la misma en el entorno estudiantil (percibido por $7 \%$ de la muestra). Solo el $3 \%$ visualiza la importancia de esta tanto en su formación como en el ámbito profesional. Un $7 \%$ de la muestra considera que la ética no es importante en ninguno de los dos ámbitos.

Figura 2

Porcentaje de actos antiéticos cometidos por categorías PAPA

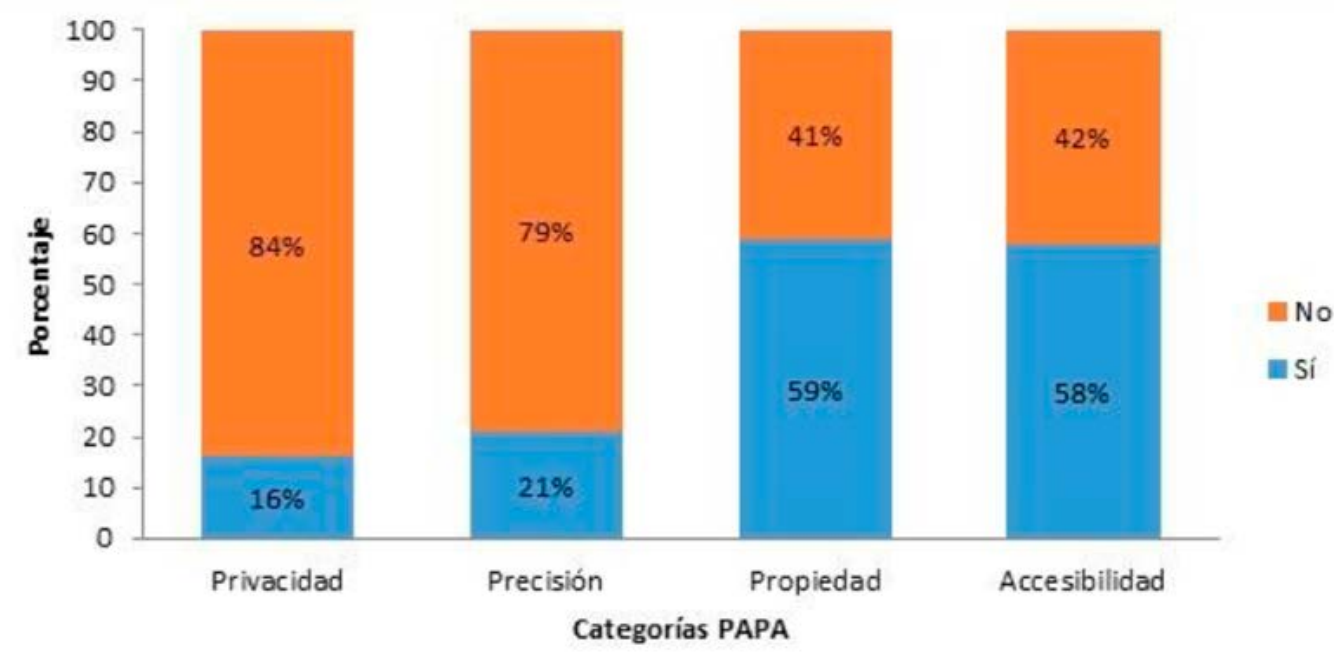

Asimismo, sobre el conocimiento respecto a las $\mathrm{TI}$, los resultados muestran que la mayoría de los participantes consideran que este es Débil (20\%), Razonable (45\%), y Bueno $(30 \%)$; pocos son los que consideran que este es Muy bueno (2\%) o Excelente (3\%). 


\section{Discusión}

El objetivo de la presente investigación es describir los procesos de razonamiento moral empleados por estudiantes de Ingeniería de Sistemas para la resolución de dilemas éticos.

Para llegar a este objetivo, en primer lugar, se analizaron las respuestas dadas a los diferentes dilemas morales planteados. Los resultados muestran que la calificación que los participantes otorgaron a los escenarios por categoría osciló entre la ambigüedad (lo cuestionable, para los escenarios de las categorías Privacidad y Propiedad), y la desaprobación (lo antiético, para los escenarios para Precisión y Accesibilidad). Estos resultados son discutidos a continuación, contrastándolos con los del estudio realizado por Martin y Woodward (2011), quienes aplicaron una versión anterior del test, similar a la actual, a dos muestras de participantes, una en Estados Unidos y otra en Europa. Se puede, entonces, realizar un análisis comparativo entre los resultados de las distintas muestras de participantes.

En la categoría de Privacidad, las tres muestras comparadas califican a los escenarios de esta categoría como cuestionables. Los participantes coinciden en el tipo de escenarios que son calificados así, en su mayoría relacionados con políticas de privacidad empresarial. Para la categoría de Precisión, nuevamente, las calificaciones de las dos muestras del estudio de Martin y Woodward (2011) coinciden con la de la muestra del presente estudio: calificaron a los escenarios propuestos como antiéticos, relacionados en esta categoría, en su mayoría, con temáticas sobre la integridad de bases de datos. Para la categoría de Propiedad, la calificación de las muestras difiere: las muestras ecuatoriana y europea coinciden y ubican a los escenarios en términos de cuestionables, mientras la muestra estadounidense calificó estos escenarios como antiéticos; y es en esta categoría donde más varían las calificaciones entre escenarios. Finalmente, para la categoría de Accesibilidad, la muestra ecuatoriana demuestra mayor desaprobación que sus contrapartes, calificando en promedio a los escenarios como antiéticos, mientras las muestras europea y estadounidense lo hacen como cuestionables: varios escenarios coinciden en su calificación y se refieren en su mayoría al uso de recursos más allá de aquello para lo que fueron planificados. Las varias coincidencias encontradas en todas las categorías muestran que el razonamiento moral de la muestra ecuatoriana es similar al de personas de otros lugares, incluso con referentes culturales muy diferentes. En general, además, se puede observar que ninguna de las tres muestras obtuvo resultados que ubiquen a los escenarios en niveles éticos o aceptables; el razonamiento moral de los individuos es entonces adecuado, ya que ninguno de los escenarios propuestos en el test es ético per se. Sin embargo, tampoco ninguna de las muestras llega a calificar a los escenarios como delictivos, aunque muchos de ellos son considerados delitos en las sociedades de origen de los participantes. Respecto a este punto, quedan por explorar entre los participantes las discrepancias entre su ética individual y la social.

En segundo lugar, en cuanto al tipo de razonamiento moral que subyace a las calificaciones de los dilemas morales, los resultados obtenidos demuestran que existe un predominio por el razonamiento emocional: todas las categorías rebasaron el $60 \%$ de respuestas para este tipo de razonamiento. Esto puede explicarse a través de la teoría de Greene (2014), para quien el proceso de tipo emocional funciona asociado a respuestas automáticas, rápidas y estereotipadas; esta es la modalidad usual del cerebro humano, en la que prevalece la eficacia conductual al preferir reacciones inmediatas al estímulo, de poco esfuerzo a la memoria de trabajo, y que habitualmente se las experimenta sin mayor conciencia (Greene, 2014a). Este tipo de respuesta va ligado a razonamientos característicamente deontológicos, relacionados a la moralidad de los derechos y deberes: se realizan en búsqueda del respeto hacia determinadas reglas tomadas como inquebrantables, independientemente de los involucrados o el contexto (Greene, 2007). Es el caso de los participantes de este estudio, quienes responden de manera emocional, posiblemente aplicando reglas fundamentales que han interiorizado de manera rápida para resolver los dilemas que se les presentó en esta investigación.

Adicional a las propuestas de resolución de dilemas y la justificación del razonamiento que las subyace, el presente estudio solicitó a los participantes reportar si habían realizado algunas conductas antiéticas, lo que añade una medición de comportamiento. Los anteriores apartados permitieron concluir que los participantes, cognitivamente, optaban por resoluciones sobre los mencionados conflictos entre la duda y la desaprobación, y lo hacían basándose en la consideración de derechos y/o deberes. No obstante, esta inclinación cognitiva no coincide del todo con los resultados comportamentales obtenidos. Así, aunque cognitivamente los participantes ubican los escenarios descritos entre cuestionables y antiéticos-lo cual demuestra un buen razonamiento moral-, ya confrontados con lo que ellos han ejecutado, observamos que entre $16 \%$ y $59 \%$ de la muestra reporta participar en actos antiéticos. Las dos categorías en donde es más marcada la diferencia son: Accesibilidad-que se cuestiona por las condiciones y características del acceso a la información-y Propiedad-que se refiere, sobre todo, a derechos intelectuales (Mason, 1986; Woodward et al., 2011). Aunque los escenarios de estas categorías fueron calificados respectivamente como antiéticos y cuestionables, ambas categorías reportan 
participación de casi el $60 \%$ en hechos antiéticos. Es interesante ver entonces que estos temas no parecen plantear mayor conflicto; no es así para la categoría de Privacidad, que demuestra más consistencia entre lo que los participantes piensan al respecto (los escenarios son cuestionables) y lo que han hecho (solo el $16 \%$ ha cometido actos antiéticos). La categoría de Privacidad reflexiona respecto a qué información puede un individuo decidir mantener privada o no y bajo qué condiciones (Mason, 1986; Woodward et al., 2011). El tema de Privacidad, de alguna manera, parece ser percibido como más antiético que los otros, ya que en él se incurre menos en actos antiéticos: puede deberse al énfasis que socialmente se hace en ello, o incluso a la presencia de leyes más estrictas para los delitos que atentan contra la privacidad. Independientemente de lo que esté detrás de estas discrepancias, en general esta comparación evidencia la problemática relación entre cognición y conducta, que ya ha sido señalada en la literatura: lo que una persona piensa respecto a una situación no se traduce necesariamente en un comportamiento que va en la misma dirección (Ajzen y Fishbein, 2005).

En última instancia, frente a la pregunta sobre la importancia de la ética, los resultados obtenidos muestran que para los participantes esta es importante a nivel profesional, no así en el momento en el que se encuentran. Además, aunque cursan la carrera de Ingeniería de Sistemas, los estudiantes declaran tener conocimientos predominantemente medio-bajos respecto a las TI. Estas apreciaciones son esenciales porque ponen en contexto los resultados anteriores. Los participantes de esta investigación, al ser estudiantes, no visualizan a los dilemas éticos como algo en lo que deban pensar en este momento: los ven como asuntos para preocuparse más tarde, cuando ya ejerzan su profesión; de esta manera, el incurrir en conductas antiéticas no les ha planteado conflicto puesto que las han realizado fuera del actuar profesional. En parte, esto puede deberse a su falta de conocimiento sobre las $\mathrm{TI}$ y las implicaciones éticas que estas tienen. Existe entonces una asociación entre la ética y el ejercicio profesional, y concomitantemente una falta de relación entre la ética personal y la profesional. Este es un ámbito de alto interés para la formación de futuros profesionales: se debería enfatizar a nivel de sus estudios el ligar la ética profesional y la ética personal, subrayando que no son cosas aisladas o diferentes.

Respecto a la investigación realizada se finaliza con determinadas reflexiones. Primeramente, es pertinente enfatizar que se trata de un estudio pionero: es la primera vez que se realiza una investigación de esta índole con una muestra ecuatoriana, y a través de un instrumento también pionero (Harris, 2017), en constante revisión. Un aporte novedoso es el haber ligado el tema de la ética en las $\mathrm{TI}$ con el del razonamiento moral en psicología, siendo que la literatura existente aborda el tema de las categorías PAPA relacionándolas con los campos de la tecnología informática y la ética empresarial (Ming et al., 2015). Sin embargo, se trata de un estudio preliminar, con datos obtenidos a través de una muestra muy específica. Debido a esto, se invita a que futuras investigaciones en el mismo campo y/o con temáticas similares puedan corroborar o refutar los hallazgos aquí encontrados. Se sugieren principalmente el uso de otros instrumentos o metodologías alternativas, así como posibles variaciones en el aspecto teórico-otras propuestas sobre el razonamiento moral, así como otras propuestas sobre el abordaje de los conflictos éticos relacionados a las TI-. Este tipo de investigaciones tienen cada vez más importancia debido a la creciente relevancia que poseen las $\mathrm{Tl}$ y los cuestionamientos éticos que suponen a los profesionales que trabajan con ellas, por lo que se debería también investigar con muestras de profesionales. Igualmente, se sugiere en futuras investigaciones profundizar respecto a una de las grandes problemáticas para la psicología cognitiva, evidenciada en esta investigación: las diferencias entre cognición y conducta en los asuntos morales.

\section{Referencias}

1. Ajzen, I. \& Fishbein, M. (2005). The influence of attitudes on behavior. In D. Albarracín, B. T. Johnson, \& M. P. Zanna (Eds.), The handbook of attitudes (pp. 173-221). Mahwah, NJ: Erlbaum. https://doi.org/10.4135/9781446214299.n4

2. Brown, K. C. (2018). A consideration of Mason's ethical framework. The importance of PAPA factors in the 21st century: A seven-year study. UNF Graduate Theses and Dissertations.

3. Bucciarelli, M., Khemlani, S., \& Johnson-Laird, P. (2008). The psychology of moral reasoning. Judgment and Decision Making, 3, 121-139.

4. Dubé, J. É. (2008). Evaluación del acuerdo interjueces en investigacion clinica. Breve introducción a la confiabilidad interjueces. Revista Argentina de Clínica Psicológica, 17, 75-80.

5. Evans, J. S., \& Stanovich, K. E. (2013). Dual-process theories of higher cognition: Advancing the debate. Perspectives on Psychological Science, 8, 223-241. https://doi. org/10.1177/1745691612460685 
6. Greene, J. D. (2007). The secret joke of Kant's soul. En W. Sinnott-Armstrong, Moral Psychology (Vols. 3): The Neuroscience of Morality: Emotion, Disease, and Development, págs. 35-116). Cambridge, MA: MIT Press.

7. Greene, J. D. (2014a). Beyond point-and-shoot morality: Why cognitive (neuro)science matters for ethics. Ethics, 124, 695-726. https://doi.org/10.1086/675875

8. Greene, J. D. (2014b). The cognitive neuroscience of moral judgment and decision making. En M. Gazzaniga, \& G. Mangun, The cognitive neurosciences (pp. 1013-1023). Cambridge, MA: MIT Press.

9. Greene, J. D. (2015). The rise of moral cognition. Cognition, 135, 39-42. https://doi.org/10.1016/j. cognition.2014.11.018

10. Greene, J. D., Nystrom, L. E., Engell, A. D., Darley, J. M., \& Cohen, J. D. (2004). The neural bases of cognitive conflict and control in moral judgement. Neuron, 44, 389-400. https://doi. org/10.1016/j.neuron.2004.09.027

11. Greene, J. D., Sommerville, R. B., Nystrom, L. E., Darley, J. M., \& Cohen, J. D. (2001). An fMRI investigation of emotional engagement in moral judgment. Science, 293, 2105-2108. https://doi. org/10.1126/science.1062872

12. Harris, A. (2017). Ethical IT Survey.

13. Harris, A., Yang, M., Yates, D., \& Kruck, S. (2011). Incorporating ethics and social responsibility in IS education . Journal of Information Systems Education 22, 183-189.

14. Hersh, R. H., Reimer, J., \& Paolitto, D. P. (2002). El crecimiento moral de Piaget a Kohlberg. Madrid: Narcea S. A.

15. Kohlberg, L. (1976). Moral stages and moralization: The cognitive devolpmental approach. En R. a. Holt, Moral devolpment and behavior: theory, research and social issues (págs. 34-35). Nueva York: S.E.

16. Martin, N. L., \& Woodward, B. (2011). Computer ethics of american and european Information Technology students: a cross-cultural comparison. Issues in Information Systems, 12, 78-87. https://doi.org/10.48009/1 iis 2011 78-87

17. Mason, R. (1986). Four ethical issues of the information age. Management Information Systems Quarterly, 10, 5-12.

18. Ming, T. M., Jabar, M. A., Sidi, F., \& Wei, K. T. (2015). A systematic literature review of computer ethics issues. Journal of Theoretical \& Applied Information Technology, 78, 360-372.

19. Peslak, A. R. (2006). PAPA revisited: A current empirical study of the Mason framework. Journal of Computer Information Systems 46, 117-123.

20. Piaget, J. (1935). El juicio moral en el niño. Madrid: Beltrán.

21. Reynolds, G. W. (2014). Ethics in Information Technology. Boston, MA: Cengage Learning.

22. Schultz, R. A. (2006). Contemporary issues in ethics and Information Technology. London: IRM Press.

23. Stahl, B. C., \& Rogers, S. (2013). Ethical issues of emerging ICT applications -A Euro-Landscape. En J. Weckert, R. Lucas, \& M. J. Selgelid, Professionalism in the Information and Communication Technology Industry (pp. 321-341). ANU Press.

24. Stahl, B. C., Timmermans, J., \& Flick, C. (2017). Ethics of emerging Information and Communication Technologies. Science and Public Policy, 369-381. https://doi.org/10.1093/scipol/scw069

25. Sullins, J. (2018). Information Technology and moral values. (E. N. Zalta, Ed.) The Standford Encyclopedia of Philosophy. Obtenido de https://plato.stanford.edu/archives/win2018/entries/ it-moral-values/

26. Woodward, B., Martin, N., \& Imboden, T. (2011). Expansion and validation of the PAPA framework. Information Systems Education Journal (ISEDJ), 9, 28-34.

Anexo dilemas (Greene, Sommerville, Nystrom, Darley, \& Cohen, 2001; Greene, Nystrom, Engell, Darley, \& Cohen, 2004).

Dilema del tranvía: un tranvía corre sin freno en su riel, y en su camino se encuentran cinco personas que morirán de seguir este su curso; no obstante, existe una palanca que, de ser activada, desviaría al tranvía hacia un riel alternativo, donde mataría a una persona en vez de cinco, ¿activaría usted la palanca?

Dilema del puente: un puente cruza por encima del tranvía, y la única manera de detener el curso de este hacia las cinco personas, es empujando a un peatón desde el puente, cuyo impacto con el tranvía prevendría el impacto contra las cinco personas, ¿empujaría usted al peatón? 
RECIBIDO: 9 de julio de 2020 MODIFICADO: 22 de octubre de 2020

ACEPTADO: 16 de diciembre de 2020 